\title{
Cigarette smoking, alcohol intake and the risk of diabetes mellitus in Gombe State, northeast Nigeria
}

\author{
Sule J. Bathna, ${ }^{1}$ Jacob A. Dunga, ${ }^{2}$ Nura H. Alkali, ${ }^{2}$ Jafiada J. Musa, ${ }^{2}$ Abubakar A. Gombe, ${ }^{1}$ \\ Sulaiman Y. Yusuf, ${ }^{3}$ Ojobi Joseph, ${ }^{4}$ Shamaki R. Baba ${ }^{1}$ \\ ${ }^{1}$ Federal Teaching Hospital Gombe; ${ }^{2}$ Abubakar Tafawa Balewa Teaching Hospital Bauchi; \\ ${ }^{3}$ Federal Medical Center Yola, Adamawa State; ${ }^{4}$ Federal Medical Center Makurdi, Benue, Nigeria
}

\begin{abstract}
Alcohol is consumed in almost all countries worldwide. Heavy drinking can reduce the body's sensitivity to insulin, which can trigger type 2 diabetes mellitus. Diabetes can complicate chronic pancreatitis, which is overwhelmingly caused by heavy alcohol drinking. Alcohol is also high in calories, with heavy drinkers liable to obesity and a higher risk of type 2 diabetes mellitus. Similarly, cigarette smoking is associated with increased risk of type 2 diabetes mellitus among both middle-aged and elderly men and women. Nigeria is one of the three largest tobacco markets in Africa with a population of almost 13 million smokers. Northeast Nigeria ranks $3^{\text {rd }}$ among the six geopolitical zones, with a smoking rate of $6.1 \%$. In this study, we evaluated the risks of diabetes mellitus associated with alcohol consumption and cigarette smoking in Gombe State, Northeast Nigeria. This cross-sectional survey was conducted in a two-stage cluster sampling scheme based on existing administrative divisions. Study subjects were males and females aged 16 years and older who resided in the Jekadafari Ward of Gombe Metropolis. Subjects were excluded from this study if they were less than 16 years of age, pregnant women or suffered chronic conditions such as chronic kidney disease, chronic liver disease or chronic lung dis-
\end{abstract}

Correspondence: Jacob A. Dunga, Department of Internal Medicine, Pulmonology Unit, Abubakar Tafawa Balewa University Teaching Hospital, Bauchi, Nigeria.

Tel.: +234.08032306016.

E-mail: jacobdunga@yahoo.com

Key words: Diabetes Mellitus alcohol, Cigarette smoking, Nigeria.

Contributions: the authors contributed equally.

Conflict of interest: the authors declare no potential conflict of interest.

Funding: none.

Received for publication: 1 April 2019.

Revision received: 15 June 2019.

Accepted for publication: 15 June 2019.

This work is licensed under a Creative Commons Attribution NonCommercial 4.0 License (CC BY-NC 4.0).

(C) Copyright: the Author(s), 2019

Licensee PAGEPress, Italy

Annals of African Medical Research 2019; 2:71

doi:10.4081/aamr.2019.71 eases based on clinical history and laboratory results. The calculated sample size was 1600 based on a $3.1 \%$ prevalence rate of DM, with a power of $85 \%$ and precision of $5 \%$. We obtained complete data on 1302 subjects, of whom $50(3.8 \%)$ had DM. (5.1\% in males and $2.86 \%$ in females). Alcohol use was prevalent among $365(28.03 \%)$ subjects, and was higher in males (49.4\%) compared to females (11.4\%). The risk of DM was significantly higher among alcoholusers compared to non-users, with an odds ratio of 4.1 (95\%CI: $2.3-$ 7.3; $\mathrm{P}=0.0001)$. Eighty-eight subjects were cigarette smokers, of whom 87 were males and only one was female. We found no significant association between cigarette smoking and $\mathrm{DM}(\mathrm{OR}=0.34$, $95 \%$ CI $0.05-2.48 ; \mathrm{P}=0.29$ ). The overall prevalence of $\mathrm{DM}$ was $3.9 \%$. Alcohol consumption, but not cigarette smoking, was associated with a higher risk of DM in this study.

\section{Introduction}

The prevalence of diabetes and its risk factors is increasing worldwide. It appears to be an epidemic affecting an increasing proportion of populations in many parts of the world. ${ }^{1}$ The global prevalence of diabetes mellitus is estimated to rise from 135 million in 1995 to 300 million by 2025 (5.4\% of the world population) with a $27 \%$ increase in developed countries and a $48 \%$ increase in developing countries. ${ }^{1}$ Globally, an estimated 422 million adults were living with diabetes in 2014, compared to 108 million in 1980. The global prevalence (age-standardized) of diabetes has nearly doubled since 1980 , rising from $4.7 \%$ to $8.5 \%$ in the adult population. This reflects an increase in associated risk factors such as alcohol consumption, cigarette smoking, overweight and obesity.

Over the past decade, diabetes prevalence has risen faster in low- and middle-income countries than in high-income countries. Diabetes caused 1.5 million deaths in 2012. Higher-than-optimal blood glucose caused an additional 2.2 million deaths, by increasing the risks of cardiovascular and other diseases. Forty-three percent of these 3.7 million deaths occur before the age of 70 years. The percentage of deaths attributable to high blood glucose or diabetes that occurs prior to age 70 is higher in low- and middleincome countries than in high-income countries. ${ }^{2}$

In Africa, diabetes was considered rare in the last century. Population-based reports of diabetes mellitus in Africa are few but the WHO estimates the prevalence of DM in Sub-Saharan Africa to be in the range of $1-2 \%{ }^{2}$

Alcohol is consumed in almost all countries of the world. Belarus has the highest per capital alcohol consumption (17.5 per capita). ${ }^{3}$ Nigeria standing at $41^{\text {st }}$ position has a projected 11.3 per capita by 2015 , about $46.8 \%$ beer, $36.4 \%$ wine and $16.9 \%$ spirit. ${ }^{1}$ Binge drinking and high alcohol consumption may increase the risk of type 2 diabetes in both women and men. ${ }^{4-7}$ Heavy drinking can reduce the body's sensitivity to insulin, which can trigger type 
2 diabetes. ${ }^{8}$ Diabetes is a common side effect of chronic pancreatitis, which is overwhelmingly caused by heavy drinking. Alcohol is also high in calories, which promotes overweight and a higher risk of type 2 diabetes. ${ }^{9}$ Similarly, cigarette smoking is associated with increased risk of type 2 diabetes mellitus among both middle-aged and elderly men and women. ${ }^{10-12}$

Nigeria is one of the three largest tobacco markets in Africa, others being Egypt and South Africa. Tobacco sales in Nigeria have continued for long with profits of the Nigerian tobacco companies increasing yearly. Although there are no records of consumers in Nigeria, it is estimated that Nigeria has a population of almost 13 million smokers, and 18 billion cigarettes are sold each year at a value of about $\$ 931 \mathrm{~m}$ (N185 billion naira). North east of Nigeria ranks $3^{\text {rd }}$ among the three geopolitical zones with a smoking rate of $6.1 \% .^{13}$

\section{Materials and Methods}

This study was conducted in Gombe, the capital of Gombe State in Northeast Nigeria. It is an urban town with rapid industrialization and influx of people from all over the state. The state has a population of over two million people based on the 2006 census. The site of the study was Jekadafari Ward in Gombe Metropolis with a current population of 268,000 . Jekadafari was randomly selected using a two-stage cluster sampling scheme based on existing administrative divisions. The ward population is composed of mainly unskilled laborers, farmers, petty traders, teachers, civil servants, businessmen and few fishermen. Alcohol and cigarettes is fairly consumed by the inhabitants of this ward.

During sampling the outcome variable was considered as the presence of DM based on the World health Organization (WHO) diagnostic criteria. ${ }^{2}$ while the exposure variables were alcohol and cigarette consumption. Possible cofounders to the study are drugs, presence of retroviral infection, pancreatic disease and chronic liver disease. The calculated sample size was 1600 based on prevalence of DM (3.1\%) with a power of $85 \%$ and precision of $5 \%$.

Subjects were male and female residents of Jekadafari aged 16 years and above who consented to the study. Subjects were excluded from the study if they were less than 16 years, pregnant or had chronic disease conditions such as chronic kidney disease, chronic liver disease or chronic lung diseases based on history laboratory investigations.

Data was analyzed using the SPSS 21 statistical programme. Means $( \pm \mathrm{SD})$ were used to describe continuous variables and proportions were used for categorical data. Two-tailed student's t-test was used for comparisons of group means. When comparing groups of subjects, the chi-squared $\left(\mathrm{X}^{2}\right)$ test was applied to determine the significance of the differences observed.

\section{Results}

Of the sample size of 1600 enlisted during the household census, 298 people did not turn up during the survey; therefore, the analysis was based on 1302 subjects giving a response rate of $81.4 \%$.

\section{Socio-demographic and clinical characteristics of study subjects}

\section{Age and sex distributions}

The mean (SD) ages of males and females were 35.31 (14.96) and 37.68 (14.44) years respectively. The age ranges are shown in Table 1 .

\section{Alcohol intake}

The distribution of subjects by alcohol use is shown in Table 2 . We obtained complete data on 1302 subjects, of whom $50(3.8 \%)$ had DM (5.1\% in males and $2.86 \%$ in females). Alcohol use was prevalent among $365(28.03 \%)$ subjects, and was higher in males (49.4\%) compared to females (11.4\%). The risk of DM was significantly higher among alcohol-users compared to non-users, with an odds ratio of 4.1 (95\% CI: $2.3-7.3 ; P=0.0001)$.

\section{Cigarette smoking}

The distribution of subjects by history of cigarette smoking is shown in Table 3. Eighty-eight subjects were cigarette smokers, of whom 87 were males and only one was female. We found no significant association between cigarette smoking and $\mathrm{DM}(\mathrm{OR}=0.34$, 95\% CI 0.05-2.48; $P=0.29$ ).

\section{Discussion}

This study has investigated the risk of DM associated with alcohol and cigarette smoking in an urban community of Gombe State, Northeast Nigeria. We found a DM prevalence rate of $3.9 \%$ (5.7\% in males and $2.8 \%$ in females), which is higher than the rates previously reported in other population surveys in Nigeria. ${ }^{14}$

Screening for DM by measuring casual blood glucose levels is an accepted method for obtaining a crude estimate of the prevalence of diabetes in a population, and it is less expensive than the standard WHO method (fasting and 2 hours post $75 \mathrm{~g}$ glucose load). ${ }^{15,16}$ Application of the standard criteria for the confirmation of DM is desirable but often impracticable under field conditions especially in areas with very scarce resources. ${ }^{15}$ The findings from this study, therefore, provides a crude estimate of the prevalence rates of DM among adults in Gombe Metropolis.

The younger age study of participants could also be a factor as about $73 \%$ of subjects fall between 15 to 45 years of age. Alcohol consumption is generally more prevalent among younger age groups. $^{17}$

Alcohol use was prevalent among 365 (28.03\%) subjects, and was higher in males $(49.4 \%)$ compared to females $(11.4 \%)$. The risk of DM was significantly higher among alcohol-users compared to non-users, with an odds ratio of 4.1 (95\%CI: 2.3-7.3; $\mathrm{P}=0.0001$ ).

The prevalence rate of diabetes mellitus (DM) in the study population was determined in relation to some recognized risk fac-

Table 1. Demography of the study subjects.

\begin{tabular}{lcccc} 
Age & Sex & & Total & $\%$ \\
\hline $16-25$ & 196 & 148 & 344 & 26.4 \\
$26-35$ & 144 & 226 & 370 & 28.4 \\
\hline $36-45$ & 90 & 152 & 242 & 18.6 \\
$46-55$ & 68 & 80 & 148 & 11.4 \\
\hline $56-65$ & 41 & 77 & 118 & 9.1 \\
$>65$ & 32 & 48 & 80 & 6.1 \\
\hline Total $(\%)$ & $571(43.9)$ & $731(56.1)$ & $1302(100)$ & 100 \\
\hline
\end{tabular}


Table 2. Risk of DM among subject who takes alcohol.

\begin{tabular}{|c|c|c|c|c|c|c|}
\hline \multirow{2}{*}{ Alcohol intake } & \multicolumn{2}{|c|}{ Males } & \multicolumn{2}{|c|}{ Females } & \multirow[t]{2}{*}{ Total (\%) } & \multirow[t]{2}{*}{ OR } \\
\hline & N. & DM, \% & N. & DM, \% & & \\
\hline Yes & $282(49.4)$ & 2.8 & 83 (11.4) & 1.9 & $365(28.0)$ & 1.5216 \\
\hline Never & $243(42.6)$ & 2.1 & $625(85.5)$ & 1.0 & 868 (66.7) & \\
\hline Stopped & $46(8.0)$ & 0.2 & $23(3.1)$ & & $69(5.3)$ & \\
\hline Total & \multicolumn{2}{|c|}{571} & \multicolumn{2}{|c|}{731} & $1302(100)$ & \\
\hline
\end{tabular}

Table 3. Risk of DM among subjects who smoke cigarettes.

\begin{tabular}{|c|c|c|c|c|c|c|c|}
\hline Cigarette smoking & N. & Males & $\begin{array}{c}\text { Females } \\
\text { DM, \% }\end{array}$ & $\begin{array}{c}\text { Total }(\%) \\
\text { N. }\end{array}$ & $\begin{array}{c}\text { OR } \\
\text { DM, \% }\end{array}$ & & \\
\hline Yes & 87 (15.2) & & 1.2 & $1(0.1)$ & & $88(6.8)$ & 1. 1561 \\
\hline Never & $483(84.6)$ & & 3.9 & $730(99.9)$ & 2.9 & $1213(93.1)$ & \\
\hline Stopped & $1(0.2)$ & & & $0(0)$ & & $1(0.1)$ & \\
\hline Total & & 571 & & & & $1302(100)$ & \\
\hline
\end{tabular}

tors for DM in adults. While alcohol intake was significantly associated with diabetes mellitus, cigarette smoking did not appear to be significantly associated risk factors for DM among the study subjects.

The prevalence of DM was highest in the age group 36-45 years among both sexes. It has been documented that diabetes prevalence is increasing worldwide, especially in populations transiting from traditional to modern lifestyles. ${ }^{17}$ While prevalence and incidence of type 2 diabetes are highest among adults, youths are now increasingly affected. ${ }^{18}$ This could have been the case in this study probably due to the fact that the youths were more likely to indulged in social activities like alcohol consumption and cigarette smoking. ${ }^{17}$

Alcohol use was prevalent among $28.03 \%$ study subjects, and was higher in males (49.4\%) compared to females $(11.4 \%)$. Alcohol consumption has been associated with secondary diabetes mellitus (from chronic pancreatitis and liver disease) in adults. ${ }^{4-9}$ Alcohol use was a risk factor for DM in this study, a finding similarly reported in other studies. 5,19

Smokers have 30 to $40 \%$ chance of developing type 2 DM. ${ }^{10}$ Smoking induces inflammation and oxidative stress to cause cell damage. Evidence strongly suggests that both inflammation and oxidative stress may be related to an increased risk of diabetes. ${ }^{12,20}$ Smoking is associated with a higher risk of abdominal obesity, which increases the risk of diabetes by promoting cortisol secretion. Studies have shown that smokers have higher concentrations of cortisol compared to non-smokers. ${ }^{20}$ Studies have confirmed that when people with type 2 diabetes are exposed to high levels of nicotine, insulin is less effective. People with diabetes who smoke need larger doses of insulin to control their blood sugar. Smokers with DM are also more likely to have heart and kidney disease, foot ulcers, retinopathy and peripheral neuropathy. ${ }^{20}$

However, we found no significant association between cigarette smoking and the risk of DM in this study. This is probably be due to the few numbers of smokers in the overall cohort. Variations in genetic susceptibility may also modulate the risks of DM due to alcohol use and cigarette smoking. ${ }^{21}$ It is also possible that religious and cultural factors influenced the responses of participants in this study.

\section{Conclusions}

This study has found a higher prevalence of DM compared to the national average, with alcohol consumption posing significant risk of DM. However, cigarette smoking was not significantly associated with DM. We recommend youth counselling against alcohol use and smoking as part of diabetes prevention and management strategies.

\section{References}

1. King H, Zimmet P. Trends in the prevalence and incidence of diabetes: NIDDM World Health Stat Quart 1988;41:190-6.

2. World Health Organization. Global report on Diabetes. Geneva: WHO; 2017. Available from: http://apps.who.int/iris/ bitstream/10665/204871/1/9789241565257_eng.pdf 2017

3. World Health Organization. Global status report on alcohol and health 2014. Available from: http://www.who.int/substance_abuse/publications/global_alcohol_report/en/ July 2017

4. Sofia C, Niklas H, Valdemar G, Jaakko K. Alcohol consumption and the incidence of type 2 Diabetes. Diabetes Care 2003;26:2785-90.

5. Kao LHW, Puddy IB, Boland LL, et al. Alcohol consumption and the risk of type 2 diabetes mellitus. Am J Epidemiol 2001;154:748-57.

6. Carlsson S, Hammar N, Persson PG, et al. Alcohol consumption, type 2 diabetes and impaired glucose tolerance in middleaged Swedish men. Diabet Med 2000;17:776-81.

7. Wei M, Gibbons LW, Mitchell TL, et al. Alcohol intake and incidence of type 2 diabetes in men. Diabetes Care 2000;1:1822.

8. Shah J. Alcohol decreases insulin sensitivity in healthy subjects. Alcohol Alcoholism 1987;2:103-9.

9. NHS UK. NHS Choices website. Type 2 diabetes reducing your risk. Available from: http://www.nhs.uk/Livewell/ Diabetes/Pages/Avoiddiabetes.aspx. Accessed July 2016 
10. Toshimi S, Hiroyasu I, Akio N, et al. Cigarette Smoking and Risk of Type Diabetes Mellitus among Middle-aged and Elderly Japanese Men and Women. Am J Epidemiol 2004;160: 158-62.

11. Rimm EB, Chan J, Stampfer MJ, et al. Prospective study of cigarette smoking, alcohol use and the risk of diabetes in men. BMJ 1995;310:555-9.

12. Will JC, Galuska DA, Forda ES, et al. Cigarette smoking and diabetes mellitus: evidence of a positive association from a large prospective cohort study. Int J Epidemiol 2001;30:540-6.

13. Centre for Public Policy Alternatives. A primer on Tobacco consumption and regulation in Nigeria. Available from: http://cpparesearch.org/nu-en-pl/a-primer-on-tobacco-consumption-and-regulation-in-nigeria/

14. Olatunbosun ST, Ojo PO, Fineberge NS, Bella AF. Prevalence of diabetes mellitus and impaired glucose intolerance in a group of urban adults in Nigeria. J Natl Med Assoc 1988;90:293-301.

15. Levitt NS, Katzenellenbogen JM, Bradshaw D, et al. The prevalence and identification of risk factors for NIDDM in urban Africans in Cape Town, South Africa. Diabetes Care 1993;16:601-7.
16. King H, Rewers M. On behalf of WHO Ad Hoc Diabetes reporting group. Diabetes in adults is a third world problem. WHO 1991;69:643-8.

17. Grucza RA, Bierut LJ. Cigarrete smoking and the risk for alcohol use disorders among adolescent drinkers. Alcohol Clin Exp Res 2006;30:2046-54.

18. Shaten J, Smith GD, Kuller LH, Neaton JD. Risk factors for the development of type 2 diabetes among men enrolled in the usual care group of the multiple risk factor intervention trial. Diabetes care 1993;16:1331-39.

19. Wicks ACB, Lowe RF, Jones JJ. Alcohol, a cause of diabetes in Rhodesia. South Afr Med J 1974;48:1115-7.

20. National Center for Chronic Disease Prevention and Health Promotion (US) Office on Smoking and Health. The Health consequences of smoking $50 \mathrm{yrs}$ of progress a report of the Surgeon General. Atlanta: Centers for Disease Control and Prevention; 2014.

21. Expert Committee on the Diagnosis and Classification of Diabetes Mellitus. The expert committee on the Diagnosis and classification of diabetes mellitus. Diabetes Care 2003;26: 3160-67. 